\title{
Design of Online Diagnostic System for Physical Parameters of Wind Turbine

\author{
Yujun Cai ${ }^{a}$ and Fang Yuan ${ }^{b^{*}}$
}

Jiangxi Engineering Research Center of Process and Equipment for New Energy, East China Institute of Technology, Nanchang, Jiangxi, China

a531339974@qq.com, ${ }^{\mathrm{b}} 455302348 @ q q . c o m$

* The corresponding author

\section{Keywords: Parameters; Online; Wind turbine}

\begin{abstract}
Taking the wind turbine as the research object and taking the virtual instrument as the development platform, the monitoring and fault diagnosis methods are studied, and the remote condition monitoring and fault diagnosis system design of the wind turbine is realized. The functions realized by this system are data signal acquisition, data storage, vibration signal analysis, data remote transmission, alarm system and vibration fault diagnosis, which provide a reliable basis for improving the performance of wind turbines.
\end{abstract}

\section{Introduction}

As a clean, pollution-free and renewable new energy source, wind energy is inexhaustible. It has great potential for development and broad market prospects. Therefore, wind power generation has received increasing attention from countries around the world. At present, wind power has become the fastest growing energy source in the world. Wind power generation system is an important part of the wind power generation industry. Objective and accurate measurement of wind power generation systems is a necessary condition for the development of new types of wind power generators, and also for the improvement of wind power generation. Machine performance provides a reliable basis.

At home and abroad, great progress has been made in the development level and development scale of wind energy utilization. To improve the power generation guarantee rate and power supply quality of wind power generation systems, it is necessary to conduct comprehensive monitoring and diagnosis of wind turbines. However, the monitoring and fault diagnosis system obviously lags behind the development of wind power generators. Its monitoring, analysis and diagnostic functions are still not perfect. The analysis is relatively simple and there is no effective fault diagnosis capability. Usually, the system with strong analysis function is more complicated and it is not convenient for on-site installation. It is only suitable for laboratory use. When the monitoring value of some measuring points exceeds the alarm setting value, the system can only give a warning of an early failure, and there is no way to know the type, source and specific cause of the failure. Many wind turbine generators frequently fail, which seriously affects the safe operation of wind power generators[1-4].

At present, the use of traditional general-purpose instruments is not only expensive, but also has poor coordination among instruments and instruments, and it is difficult to meet the test requirements. Therefore, it is very necessary to develop an on-line detection system for wind turbines based on virtual instrument platform. This remote monitoring and fault diagnosis system for wind turbines is a multi-functional monitoring and diagnostic analysis system integrating signal acquisition, signal analysis and fault diagnosis. It can collect and monitor the vibration, temperature and pressure parameters of the key components of the wind generator in real time, compare the monitoring results with the set alarm values to determine whether there is a fault in the equipment components; using various analysis methods to collect the data processing, with more advanced analysis technology for further diagnostic analysis, to improve the accuracy of the test process, to avoid human reading errors, improve anti-jamming capability, to minimize the error, improve the efficiency of the test, in order to facilitate the specific causes of the failure and its source makes accurate judgments. 
Wind turbine working condition monitoring and fault diagnosis system is composed of hardware and software. It relies on virtual instrument platform to realize data acquisition, analysis and fault feature extraction. First, an acceleration sensor converts the vibration signal into a charge signal, and the rotational speed of the high-speed shaft of the wind turbine is converted into a pulse signal by a rotation speed sensor, and the charge signal is converted into a voltage signal through the charge amplifier. Then through the isolation conversion module, the easily attenuated voltage signal is converted into a current signal suitable for long-distance transmission, passed through the signal cable from the wind turbine nacelle to the ground control room, and then through the isolation conversion module, the current signal is reconverted into voltage signal. The data acquisition card of the virtual instrument collects the voltage signal, transmits the signal to the computer, and the application software records the collected data, and analyzes the signal from the time domain, frequency domain and time-frequency domain for analysis and judgment by the monitoring personnel.

\section{Hardware Design}

The wind turbine's hardware system mainly includes: acceleration sensor, speed sensor, charge amplifier, signal isolation conversion module, data acquisition board, PC and so on [5-6].

\section{Selection of Vibration Acceleration Sensors}

Vibration acceleration sensors are that converts vibration signals into electrical signals. Select a piezoelectric acceleration sensor with passive, wide frequency response, high sensitivity, good linearity, strong anti-interference ability, low price, and strong compatibility with other signal conditioning instruments. Mounted on the high speed side of the gearbox, the generator torque input and the free end housing. The surface mounted on the corresponding test site can be bolted to facilitate the long-term operation of the system.

\section{Optoelectronic Speed Sensor Selection}

The photoelectric encoder is selected as the tachometer for measuring the rotational speed. The photoelectric tachometer converts the rotational speed into a digital pulse signal. The measurable maximum rotational speed is $30000 \mathrm{r} / \mathrm{min}$. It can also withstand the thrust of $30 \mathrm{~N}$ in the radial direction and $30 \mathrm{~N}$ in the axial direction. The counting accuracy is high. To meet the needs of wind turbine generator speed measurement.

\section{Selection of Charge Amplifiers}

The selected charge amplifier can be used with piezoelectric sensors of different sensitivities, with adjustable output gain, built-in high-pass and low-pass filters, and is suitable for monitoring requirements in harsh environments.

\section{Selection of Signal Isolation Conversion Module}

In wind turbine fault diagnosis, the signal transmission distance is long and the voltage signal is attenuated during long-distance transmission. Therefore, after the signal is conditioned, a set of isolation conversion modules are selected to convert the voltage signal and the photoelectric sensor rotation speed pulse signal into a current signal. After long-distance signal cables transmit signals to the ground control room, and then through a set of isolated conversion modules, the current signal is converted into the original signal and input to the data acquisition board. Isolation transmission module is used to realize voltage to current conversion, and the speed pulse signal is converted into voltage signal. In the ground control room, the current signal is converted back to voltage signal, and the current signal is converted into speed signal. Finally, multiple signal conditioning modules and power supplies are integrated into the same chassis to form a vibration signal transmitter. 


\section{Data Acquisition Card Selection and Wiring}

The data acquisition card has functions such as function acquisition signal, A/D conversion, counter, and signal generator. The NI data acquisition card can be selected to complete the collection of analog input channels and digital input channels at the same time.

Finally, each channel of the signal sensor, the charge amplifier, and the isolation conversion module is connected with a BNC shielded cable, and the data acquisition card is connected with the PC to form the overall hardware system of the fault diagnosis system.

\section{Software Design}

Develop software systems for fault diagnosis systems. The main software modules include data acquisition, data storage read, and data processing analysis modules. Data collection and storage is mainly the preparation of a data card driver to initialize the data card and drive it to work according to the set mode. At the same time, the collected data is recorded for data playback analysis. The data processing and analysis module includes a vibration signal processing and feature extraction module and a fault identification module of a BP neural network. The vibration signal processing and feature extraction module is an application-related algorithm that analyzes and processes the collected data and extracts the characteristic information of the signal to provide a basis for fault diagnosis. The fault identification module of the neural network uses the pattern recognition technology of the BP network to intelligently identify the type of the fault.

\section{Data Acquisition Module}

The data acquisition module mainly includes a data acquisition card initialization module and a data acquisition driving module. The main function of the acquisition system initialization module is to configure the acquisition system initialization parameters, including the type of acquisition data, sampling channels, and triggering methods. The data acquisition mode selects the continuous sampling mode. In the continuous acquisition mode, the data is read from the buffer of the acquisition card without any omission while the data is continuously collected.

\section{Data Storage and Reading Module}

The fault diagnosis system must have data storage and historical data reading capabilities. Fault diagnosis system as a continuous operation system, the data storage will take up a lot of hard disk space, in order to effectively use the storage space, must be a reasonable definition of the stored data. The stored data is saved in the form of binary files. In order to improve storage efficiency and reduce storage space, the fault diagnosis system does not directly record the acceleration values and units of each channel, and selects to record the voltage values read from the buffer of the data acquisition card. In the recording process, each channel is not recorded separately, but the two-dimensional array read out from the buffer of the entire capture card is recorded. At the same time in the record at the same time adding the sampling date, time and other information, convenient historical records can understand the corresponding information. The sensitivity conversion process of converting voltage values into acceleration values, and the functions of displaying each channel signal in the array separately, are defined in the history data viewing module to reduce the time for program access to data and improve storage and reading effectiveness.

\section{Data Filtering, Noise Reduction Processing and Signal Processing Module}

The natural environment of wind generators is harsh and the wind load changes irregularly. The structure itself is complex, and the vibration forms are various. In the process of work, noise and electromagnetic interference are great. The above factors have a great impact on the data collection results of the wind turbine fault diagnosis system. The signals measured under noise and 
electromagnetic interference must undergo corresponding filtering and noise reduction processing to remove the components that are not related to the vibration of the wind turbine during the measurement process. The design is from both hardware and software to suppress and remove interference noise. Improve the signal to noise ratio of the signal. The main method to eliminate interference in hardware design is shield the noise source and select a reasonable sensor layout location. Ground fault shielding and protection of the fault diagnosis system. Twisted pair shielded cables are used during signal transmission. Use the filter circuit to carry on the low pass filter processing to the analog signal, amplify the signal before the signal transmission, improve the signal to noise ratio, use the electric current signal transmission in the long distance transmission process of the signal, prevent the signal from attenuating excessively distorted. The software combines the characteristics of the wind turbine vibration signal, proposes a signal filtering method based on the digital filter and a de-noising method based on the wavelet threshold to process the signal to achieve the purpose of removing the interference signal and the noise signal.

In the fault diagnosis of wind turbines, proper signal processing and feature extraction methods can be used to quickly and accurately obtain fault information and determine the type, location, and severity of the fault. The fault diagnosis system uses parameter statistics, probability distribution statistical methods, and correlation analysis methods in the time domain, frequency spectrum and cepstrum methods in the frequency domain, and wavelet packet decomposition and frequency band energy monitoring methods in time-frequency analysis to extract vibration signals. The information and characteristics of the signal, and input the signal's feature vector into the artificial neural network, through the artificial intelligence method to obtain the auxiliary diagnosis conclusion, helps the engineering technician to carry on the fault diagnosis work.

\section{Summary}

The overall structural design of the monitoring and fault diagnosis system for wind turbines will be studied, and the selection of system hardware components will be completed. The target for monitoring the wind turbines, the selection of monitoring points and the selection of data acquisition cards will be determined; the selection of software development platforms and the interface of the system will be determined; research the development of remote monitoring and data analysis system modules for wind turbines, including the design of modules for data acquisition, storage, reading, wavelet noise reduction, etc. To realize cepstrum analysis, wavelet envelope analysis, and mapping of vibration signals. Based on the theory of wavelet and neural network, the intelligent fault diagnosis module of the generator set is designed, and the fault feature extraction of the vibration signal is accomplished by using the artificial neural network diagnosis method to realize the intelligent diagnosis of the bearing fault of the wind turbine generator. For signals that are likely to produce interference test accuracy in the analysis test and diagnosis process, different anti-jamming measures are taken to improve the test accuracy of the monitoring and diagnostic system.

\section{Acknowledgements}

The technology research project of Jiangxi education department (GJJ171509) (GJJ171510) and the open project program of Jiangxi engineering research center of process and equipment for new energy (JXNE2017-07) and 2017 Fuzhou science and technology plan project and Educational reform project in Jiangxi province (JXJG-17-37-3) and 2014 Jiangxi university students innovation and entrepreneurship project supported this work.

\section{References}

[1] El-Thalji I, Jantunen E. On the development of condition based maintenance strategy for offshore wind farm : requirement elicitation process. Energy Procedia. 24(2012)328-339. 
[2] R Matsuzaki and A Todoroki, Tokyo Institute of Technology. Wireless detection of internal delaminating cracks in CFRP laminates using oscillating frequency changes. Composites Science and Technology. 66(2006)407-416.

[3] Tavner P J,Xing J,Spinato F.Reliability analysis for wind turbines. Wind Energy. 10(2007) 1-18.

[4] Sumit K,Nath R M,Vasu M. Pandit.Wavelet based compression and denoising of optical tomography data.Optics Communications. 167(1999)37-46.

[5] Qin, Datong. Optimization Design of System Parameters of the Gear Transmission of Wind Turbine Based on Dynamics and Reliability.Chinese Journal of Mechanical Engineering. 44 (2008)24-31 .

[6] Wei, J., Lv, C., Sun, W., Li, X., \& Wang, Y. A study on optimum design method of gear transmission system for wind turbine. International Journal of Precision Engineering \& Manufacturing. 14(2013)767-778. 